Psychotherapeut 2002 $47: 315$

\section{Redaktion \\ M. Cierpka, Heidelberg}

Unter ständiger Mitarbeit

M. Stasch, Heidelberg

Psychotherapie im Internet

Claudia Neuhaus · Krefeld

\title{
Terror im Ohr: Tinnitus
}

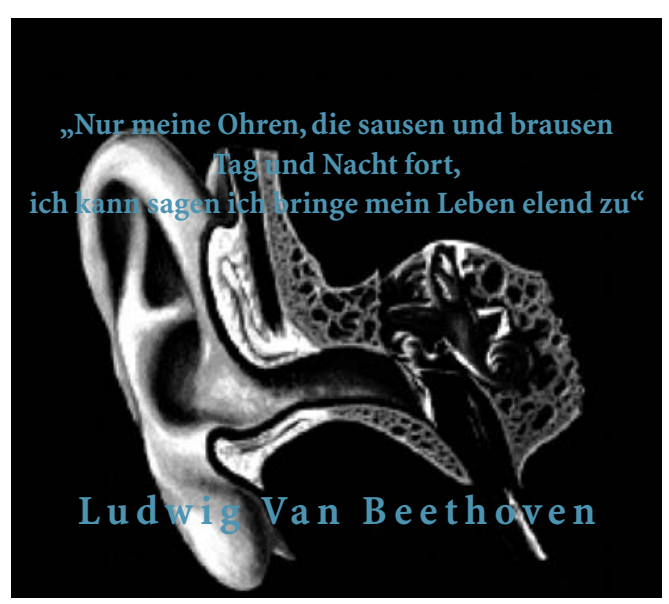

Abb. 1 Auch Beethoven

litt unter Ohrgeräuschen

(http://www.tinnitus-inti.de)

fehlerhaft arbeitet und so Signale ins Bewusstsein dringen, die normalerweise nicht wahrgenommen werden. Damit der Tinnitus nicht mehr im Zentrum der Wahrnehmungen steht, trägt der Betroffene einen „Masker“ - ähnlich einem Hörgerät -, der ein ständiges Geräusch (weißes Rauschen) produziert. So soll der Tinnitus ins Abseits gerückt und wieder "verlernt" werden. Die TRT besteht aus einem 4-Säulen-Konzept: Counseling (Beratung), psychologische Betreuung, Entspannungstechniken und Geräteversorgung. Dies setzt die interdisziplinäre Zusammenarbeit von HNOÄrzten, Psychologen, Psychotherapeuten und Geräteakustikern voraus (http:// www.ohrensausen.de/).

In der Regel wenden Tinnituszentren die TRT an, zum Teil mit psychologischer, bzw. psychotherapeutischer Exploration und gegebenenfalls Therapie (www.tinnitus-therapie-zentrum.de, www.tinnitus-inti.de). Zahlreiche $\mathrm{Be}-$ handlungsstudien und Tinnitusprojekte (http://www.zi-mannheim.de/neuropsych/lerstnp_flyer.htm) zeigen jedoch, dass die notwendige Differenzialindikation, die zu gezielten Therapiemethoden führen könnte, noch nicht geklärt ist.
Informationen über Tinnitus, Selbsthilfegruppen und Veranstaltungen für Betroffene und Experten stellt die Deutsche Tinnitus-Liga e. V. (www.tinnitus-liga.de) zur Verfügung. Eine ausgezeichnete Linkliste und umfangreiche Arztinformationen zum Thema Tinnitus finden sich unter $w w w$.forum-tinnitus.de.

Zwar werden Betroffene heute nicht mehr zur Ader gelassen, doch angesichts des Therapiedschungels kann der Patient Behandlungen erfahren, die sein Leiden verstärken und manifestieren. Die Gesellschaft für Hals-, Nasen-, Ohren-Heilkunde, Kopf- und Hals-Chirurgie hat eine Leitlinie zur Behandlung von Tinnituspatienten (http://www. uni-duesseldorf.de/WWW/AWMF/ll/hno_ll63.htm) vorgestellt, um zukünftige Therapieverfahren effektiver zu gestalten.

(C) Springer-Verlag 2002

Claudia Neuhaus

Jägerstraße 52,47798 Krefeld

neuhaus.claudia@t-online.de org). Es beruht auf der Annahme, dass die Geräuschfilterfunktion im Gehirn 